\title{
Insufficient Gestational Weight Gain and Infant Neurodevelopment at 12 Months of Age: the Japan Environment and Children's Study
}

\section{Noriko Motoki ( $\sim$ nmotoki@shinshu-u.ac.jp )}

Shinshu University Graduate School of Medicine School of Medicine: Shinshu Daigaku Daigakuin Igakukei Kenkyuka Igakubu https://orcid.org/0000-0002-6611-7426

\section{Yuji Inaba}

Shinshu University Graduate School of Medicine School of Medicine: Shinshu Daigaku Daigakuin Igakukei Kenkyuka Igakubu

\section{Takumi Shibazaki}

Shinshu University Graduate School of Medicine School of Medicine: Shinshu Daigaku Daigakuin Igakukei Kenkyuka Igakubu

\section{Yuka Misawa}

Shinshu University Graduate School of Medicine School of Medicine: Shinshu Daigaku Daigakuin Igakukei Kenkyuka Igakubu

\section{Satoshi Ohira}

Shinshu University Graduate School of Medicine School of Medicine: Shinshu Daigaku Daigakuin Igakukei Kenkyuka Igakubu

\section{Makoto Kanai}

Shinshu University Graduate School of Medicine School of Medicine: Shinshu Daigaku Daigakuin Igakukei Kenkyuka Igakubu

\section{Hiroshi Kurita}

Shinshu University Graduate School of Medicine School of Medicine: Shinshu Daigaku Daigakuin Igakukei Kenkyuka Igakubu

\section{Teruomi Tsukahara}

Shinshu University Graduate School of Medicine School of Medicine: Shinshu Daigaku Daigakuin Igakukei Kenkyuka Igakubu

\section{Tetsuo Nomiyama}

Shinshu University Graduate School of Medicine School of Medicine: Shinshu Daigaku Daigakuin Igakukei Kenkyuka Igakubu

The Japan Environment and Children's Study Group

Nagoya City University: Nagoya Shiritsu Daigaku

\section{Research Article}


Keywords: Gestational weight gain, Infant, Neurodevelopment, Developmental delay, ASQ-3

Posted Date: April 20th, 2021

DOI: https://doi.org/10.21203/rs.3.rs-435377/v1

License: (c) (1) This work is licensed under a Creative Commons Attribution 4.0 International License. Read Full License 


\section{Abstract}

\section{Background}

Abnormal gestational weight gain (GWG) increases the risk of obstetric-related complications. This investigation examined the impact of GWG on infant neurodevelopmental abnormalities at 12 months of age using the data of a nationwide Japanese cohort study.

\section{Methods}

Questionnaire data were obtained from the ongoing Japan Environment and Children's Study cohort study. GWG was subdivided as below, within, or above the reference values of the Institution of Medicine pregnancy weight guidelines. The Ages and Stages Questionnaire, third edition (ASQ-3) is a parentreported developmental screening instrument for children across five domains: communication, gross motor, fine motor, problem solving, and personal-social. Multiple logistic regression analysis was employed to identify correlations between GWG and developmental delay defined as ASQ-3 scores of less than two standard deviations below the mean.

\section{Results}

A total of 30,694 mothers with singleton live births and partners who completed the questionnaire were analyzed. The prevalence of mothers below, within, and above the GWG guidelines was $60.4 \%(18,527)$, $32.1 \%(9,850)$, and $7.5 \%(2,317)$, respectively. We recorded 10,943 infants $(35.7 \%)$ who were outliers in at least one ASQ-3 domain. After controlling for covariates, GWG below established guidelines was associated with a significantly higher risk of developmental delay for the communication (odds ratio [OR] 1.21, 95\% confidence interval [Cl] 1.09-1.34), gross motor (OR 1.14, 95\% Cl 1.05-1.24), fine motor (OR 1.13, $95 \% \mathrm{Cl} 1.04-1.24)$, problem solving (OR 1.09, 95\% Cl 1.01-1.18), and personal-social domains (OR 1.15, $95 \% \mathrm{Cl} 1.07-1.24)$.

\section{Conclusion}

This large survey revealed a possible deleterious effect of insufficient GWG on infant neurodevelopment.

The Japan Environment and Children's Study (JECS) was registered in the UMIN Clinical Trials Registry on 15 January 2018 (number: UMIN000030786).

\section{What Is Known:}

Inappropriate gestational weight gain could cause obstetric complications and adverse birth outcomes. Excess weight gain has resulted in gestational diabetes, hypertension, eclampsia, caesarean delivery, and macrosomnia. Insufficient weight gain has been associated with preterm birth and small for gestational age. 


\section{What Is New:}

This study provides important information on a possible adverse effect of insufficient gestational weight gain on offspring neurodevelopment at 12 months of age.

\section{Introduction}

Developmental delay is defined as delays in the areas of speech and language, motor, social, and cognitive development [1]. The incidence of developmental delay has increased dramatically in recent decades $[2,3]$. Although the estimated prevalence of developmental delay is generally $5-15 \%$ in pediatric populations [2-4], reported rates vary depending on the socioeconomic characteristics of the study population, case definition, and age range [5].

Excess maternal weight gain increases the risk of obstetrics complications such as gestational diabetes, hypertensive disorder of pregnancy (HDP), eclampsia, caesarean delivery, macrosomnia [6]. On the other hand, insufficient gestational weight gain (GWG) has also been associated with adverse birth outcomes, including preterm birth and small for gestational age (SGA) [7] The Institute of Medicine (IOM; now known as the National Academy of Medicine) developed GWG guidelines in 1990 and later updated them in 2009 [8]. The IOM guidelines incorporate the World Health Organization (WHO) categories of maternal body mass index (BMI) and recommend lower GWG for obese women. Japan has not formally adopted the IOM guidelines, having instead developed an original set of rules for pregnancy weight management owing to limited ethnic diversity (Supplemental table S1) [9]. The Japanese guidelines are stricter for weight gain primarily to reduce obstetric complications. One large limitation of the guidelines, however, is that they lack validation from a large national study. An emerging problem among the Japanese is an increase in underweight pregnant women [10]. Such a condition has been associated with an augmented risk of SGA [7] and possibly delayed offspring development. In Japanese women, underweight may be a larger issue than obesity.

Recent reports on the longer-term risks of maternal obesity have suggested a relationship with developmental delay in early childhood, and several epidemiologic studies have found associations between maternal obesity and various neurodevelopmental outcomes [11, 12]. In contrast, there is little evidence on the early childhood effects of maternal underweight, with none on whether excess or insufficient GWG increase the risk of offspring developmental delay in Japan. We therefore conducted a large birth cohort study with the specific objective of examining the impact of maternal GWG on early neurodevelopment.

\section{Materials And Methods}

\section{Study design, population, and settings}

The data used in this study were obtained from the Japan Environment and Children's Study (JECS), an ongoing cohort study that began in January 2011 to determine the effect of environmental factors on 
children's health. In the JECS, pregnant women were recruited between January 2011 and March 2014. The eligibility criteria for participants were: 1) residing in the study area at the time of recruitment, 2) expected delivery after August 1, 2011, and 3) capable of comprehending the Japanese language and completing the self-administered questionnaire. This study was registered in the UMIN Clinical Trials Registry (number: UMIN000030786). Details of the JECS project have been described previously [13, 14]. The JECS protocol was reviewed and approved by the Institutional Review Board on Epidemiological Studies of the Ministry of the Environment (ethical number: No. 100910001) as well as by the Ethics Committees of all participating institutions. The JECS was conducted in accordance with the Helsinki Declaration and other nationally valid regulations and guidelines. Written informed content was obtained from each participant.

The present study was based on the "jecs-an-20180131" dataset released in March 2018 containing information on 98,255 mothers who had a singleton pregnancy. Specifically, we focused on questionnaire data regarding developmental screening as self-described by mothers when their child was 12 months old. The screening tool was the Ages and Stages Questionnaire, third edition (ASQ-3) [15]. Maternal medical information, additional pregnancy details, and medical history were collected from subject medical record transcriptions for adoption as other covariates.

\section{Data collection}

Information on socioeconomic status, smoking habit of the mother and partner, and maternal alcohol consumption was collected during the second/third trimester of pregnancy (T2) by means of selfreported questionnaires. Details on a parental history of neurodevelopmental disorders, epilepsy, and mental disease were also collected from T2 questionnaires as described by the mother and partner. Maternal anthropometric data before and during pregnancy, complications and medication during pregnancy related to HDP, diabetes mellitus/gestational diabetes mellitus (DM/GDM), and neonatal information was gathered from medical record transcriptions. Prepregnancy BMI was calculated according to WHO standards as body weight $(\mathrm{kg}) /$ height $(\mathrm{m})^{2}$ and categorized as underweight $(\mathrm{BMI}<$ 18.5), normal weight (BMI 18.5-24.9), overweight (BMI 25.0-29.9), and obese (BMI 30+).

\section{Outcomes}

The main outcomes of interest were ASQ-3 domain scores. The ASQ-3 is a parent-reported initial level developmental screening instrument for children aged 12 months with 30 items in five domains: communication, gross motor, fine motor, problem solving, and personal-social. Each item describes a skill, ability, or behavior to which the parent responds "yes" (10 points), "sometimes" (5), or "not yet" (0). Parents sometimes omit items when they are unsure of how to respond or because they have concerns about their child's performance of the item. ASQ-3 scores were not calculated if there were three or more omitted items in a given domain. In the case of one or two omitted items, an adjusted total domain score was calculated by adding the averaged item score either once for one omission or twice for two 
omissions. The score calculated for each domain was categorized as normal development (above cutoff) or referral zone (score less than two standard deviations below the mean) [15].

Participants with established risk factors of developmental delay, such as neonatal asphyxia, and physical abnormality at birth including infection, respiratory distress, congenital abnormality, hearing disability, and chromosomal abnormalities, were excluded (Fig. 1). Infants with no ASQ-3 abnormalities were adopted as normal development.

\section{Exposure}

GWG in this study was subdivided as below, within, or above the reference values of the 2009 IOM guidelines widely used throughout the world. The IOM guideline ranges for total GWG based on prepregnancy BMI are as follows: $12.7-18.1 \mathrm{~kg}$ for underweight women, $11.3-15.9 \mathrm{~kg}$ for women of normal weight, $6.8-11.3 \mathrm{~kg}$ for overweight women, and 5.0-9.1 kg for obese women (Supplemental table S1).

\section{Covariates}

The covariates in our models were selected a priori based on previous literature and biologic plausibility [16-20]. We estimated the effects of GWG after adjusting for demographic data including maternal age, prepregnancy BMI, parental smoking habit, maternal drinking habit, maternal highest level of education, annual household income, parental history of neurodevelopmental disorders, epilepsy, and mental disease, as well as obstetric and medical variables such as parity, means of pregnancy, use of folic acid supplements, complications during pregnancy (including DM/GDM, HDP, and intrauterine growth restriction), means of delivery, birth weight, gender, method of feeding, and neonatal jaundice. Parental medical history of neurodevelopmental disorders included attention deficit and hyperactivity disorder, learning disability, autism, Asperger's syndrome, pervasive developmental disorder, and others. Mental disease included depression, schizophrenia, and anxiety disorder.

\section{Statistical analysis}

Differences in maternal age, prepregnancy BMI, GWG, gestational weeks, and birth weight between subjects with normal development and developmental delay were assessed by the Mann-Whitney $U$ test. We categorized all continuous and ordinal variables, such as maternal age ( $<35$ or $35+$ years), prepregnancy BMI, GWG (below, within, or above), annual household income $(<4,000,000,4,000,000$ $7,999,999$, or 8,000,000+ JPY), gestational weeks ( $<37,37+$ weeks), and birth weight $(<1,500,1,500-2499$, $2,500+g)$. Fisher's exact tests or chi-square tests were performed to compare covariates between groups stratified by category as well as by the presence of developmental delay. Additionally, differences in the scores of each domain among the three GWG groups were assessed by one-way repeated measures of analysis of variance (ANOVA) followed by post hoc (Bonferroni) testing. We employed logistic regression models to calculate adjusted ORs (ORs) and their 95\% confidence intervals (Cls). Spearman's rank correlation coefficient was used to check for multicollinearity of covariates. The variable of gestational 
weeks was excluded from the covariates because it was multicollinear with birth weight. HosmerLemeshow testing was used to assess the goodness-of-fit of the models. We also analyzed the subjects without registered fathers to evaluate for possible selection bias.

All statistical analyses were performed using SPSS statistical software version 27 (SPSS Inc., Chicago, Illinois). All tests were two-tailed, and P-value of less than 0.05 were considered to indicate statistical significance.

\section{Results}

A total of 30,694 mothers with singleton live births and partners who completed the JECS questionnaire were available for analysis (Fig. 1). According the prepregnancy BMI categories, the prevalence of underweight, normal weight, overweight, and obese mothers was $15.4 \%(4,730), 74.2 \%(22,761), 8.1 \%$ $(2,485), 2.3 \%$ (718), respectively. The prevalence of mothers below, within, and above the IOM-based GWG guidelines was $60.4 \%(18,527), 32.1 \%(9,850)$, and $7.5 \%(2,317)$, respectively. There were 10,943 participants (35.7\%) who were outliers in at least one ASQ-3 domain (Table 1 ).

Table 1 summarizes the participants' characteristics and offspring outcomes for developmental delay. There were significant differences in the rates of the GWG groups. We observed significant differences between the normal development and developmental delay groups for demographic categories including maternal age, maternal educational level, annual household income, parental smoking status, and maternal history of epilepsy. Significant differences were also seen in such perinatal categories as parity, means of pregnancy of current birth, maternal use of folic acid supplements, HDP, mode of delivery, gestational weeks, birth weight, gender, method of feeding, and neonatal jaundice.

ASQ-3 domain classifications and proportions of a risk of developmental delay at 12 months according to maternal GWG are shown in Table 2. Chi-square analysis revealed significant differences in the prevalence of developmental delay in the communication, gross motor, fine motor, problem solving, and personal-social domains among maternal GWG groups. ANOVA showed that the scores for every ASQ-3 domain were significantly lower in the GWG below guidelines group than in the GWG within and above guidelines groups.

The regression models for all domains demonstrated good fit in Hosmer-Lemeshow testing. In multivariate logistic regression analysis after adjustment for covariates, compared with ideal GWG, GWG below guidelines was significantly associated with a higher incidence of developmental delay in the communication (OR 1.21, 95\% Cl 1.09-1.34), gross motor (OR 1.14, 95\% Cl 1.05-1.24), fine motor (OR 1.13, 95\% $\mathrm{Cl} 1.04-1.24)$, problem solving (OR 1.09, 95\% Cl 1.01-1.18), and personal-social (OR 1.15, 95\% Cl 1.07-1.24) domains (Table 3). For every $2.3 \mathrm{~kg} \mathrm{(5} \mathrm{lb)} \mathrm{of} \mathrm{GWG,} \mathrm{the} \mathrm{risk} \mathrm{of} \mathrm{abnormalities} \mathrm{was} \mathrm{reduced} \mathrm{by} 4$ to $9 \%$ in each domain of ASQ-3 (communication: OR 0.91 [95\% Cl 0.88-0.94], gross motor: OR 0.96 [95\% $\mathrm{Cl}$ 0.94-0.98], fine motor: OR 0.94 [95\% $\mathrm{Cl} 0.91-0.96]$, problem solving: OR 0.95 [0.93-0.97], personal-social: OR 0.94 [0.92-0.96]) (Table 3). 
Across BMI categories, GWG below guidelines tended to associate with a higher risk of developmental delay (i.e., OR > 1.0) in ASQ-3 screening than did GWG within guidelines (Fig. 2A). In contrast, GWG above guidelines often associated with a lower risk of developmental delay across domains as compared with GWG within guidelines (Fig. 2B).

Lastly, we analyzed the 24,823 subjects without registered fathers. Supplemental table S2 shows the characteristics of the normal development and developmental delay groups. We observed a significant difference in the proportion of GWG categories between the groups similar to that in the main analysis (Supplemental table S2). Multivariate regression analysis also revealed significant associations between GWG below guidelines and the incidence of developmental delay in all five domains. For every $2.3 \mathrm{~kg}$ ( 5 lb) of GWG, the risk of abnormalities was reduced by $5 \%-11 \%$ in each domain of ASQ-3 (Supplemental table S3).

\section{Discussion}

We herein describe the first large-scale nationwide birth cohort study in Japan to clarify the impact of maternal insufficient weight gain on offspring neurodevelopment at 12 months. Across prepregnancy $\mathrm{BMI}$ categories, the association was particularly significant in mothers with lower prepregnancy BMI.

In this Japanese nationwide birth cohort study, the prevalence rate of developmental delay measured by ASQ-3 at 12 months of age for communication, gross motor, fine motor, problem solving, and personalsocial domains was $6.8 \%, 13.5 \%, 9.7 \%, 15.0 \%$, and $16.5 \%$, respectively. However, global frequencies can differ for demographic status and underlying disease [4, 5, 16-18]. Knowing the risk factors of developmental delay in early childhood will facilitate the monitoring of suspected cases. Several perinatal risk factors of developmental delay have been reported, including preterm birth, perinatal maternal mental health, and maternal educational level [16-18]. Relationships between maternal obesity during pregnancy and poor pregnancy results have also been described $[6,7,11,12]$. The increasing number of obese pregnant women has become a concern in many countries. On the other hand, the number of underweight pregnant women in Japan is on the rise [10,21,22]. The obesity classification and GWG recommendations used in Japan differ considerably from those prescribed by the IOM (Supplemental table S1) [9]. Several recent Japanese studies showed that underweight women carried a higher risk of adverse birth outcomes, such as preterm birth and SGA $[21,22,23]$. However, they did not assess subsequent neurodevelopment in infants of underweight women. A Swedish cohort study investigating the association between maternal GWG and risk of offspring autism spectrum disorder (ASD) supported our findings, whereby an elevated risk of ASD was observed for both insufficient and excess GWG [24]. They also suggested that maternal undernutrition during pregnancy contributed to the risk developmental abnormality. The prevalence of insufficient GWG in Asian countries is higher than Western countries [7]. On the other hand, obesity of pregnant women has been decreasing in some developed countries in recent years $[25,26]$, and underweight of pregnant women may be a widely concerned problem. 
It is uncertain why insufficient GWG may cause neurodevelopmental disorders. One reason is that malnutrition may restrict fetal brain growth. In Japan, total calorie intake among pregnant women was far below nationally recommended levels $[27,28]$. Moreover, among underweight pregnant women, the dietary intake of protein, iron, magnesium, and folic acid was lower than that of normal weight and overweight women [28]. Maternal dietary quality is of critical importance since specific nutrients are required during sensitive or critical periods of fetal development [29]. Folic acid and choline have been recognized as necessary for neural tube development [30-32]. Zinc has important roles in neuron formation, migration, and synapse generation [33]. Furthermore, pregnant vegetarian women are at risk for vitamin B12 deficiency associated with delayed myelination [31]. Iron is the most common nutrient deficiency during pregnancy and is necessary for myelination and the development of the frontal cortex and basal ganglia [34].

The studies on Japanese pregnant women mentioned above reported that the proportions of carbohydrates and lipids in total calories were respectively lower and higher than those required by pregnant women $[27,28]$. Sussman et al. suggested that prenatal exposure to a carbohydrate-restricted diet, such as recently-popular ketogenic diet programs, influenced offspring neuro-anatomy and behavior $[35,36]$. Assessment of the brain structure of neonate mice whose mothers were fed a ketogenic diet also revealed several major structural and volumetric differences [35]. Furthermore, prenatal exposure to a ketogenic diet resulted in behavioral alterations that included reduced susceptibility to anxiety and depression and elevated hyperactivity in adult mouse offspring [36]. Considering the neurodevelopmental prognosis of children, optimal diet and weight gain guidance for underweight women of child-bearing age is critical.

We investigated the relationship between offspring development at 12 months and maternal GWG; however, it is important whether the evaluations at 12 months are clinically valid for subsequent diagnosis. In one study longitudinally comparing child ASQ-3 domain screening results based on cut-off scores, the vast majority (88.9\%-96.7\%) received the same categorization results at 9,18 , and 24 months of age [37]. Other studies have provided evidence on the concurrent validity of the ASQ and the clinical diagnosis of developmental delay [38-40]. Furthermore, they also showed the reliability of the ASQ-3 in a multi-ethnic population [38-40]. As the present study cohort will be followed until the age of 13 years, further investigation on the association of ASQ-3 screening with clinical diagnosis is warranted.

A strength of this investigation was that not only maternal, but also paternal history of neurodevelopmental problems, such as mental disease, developmental disorder, and epilepsy, were adjusted for as covariates. Genetic influences could be larger than those of a shared environment on the incidence of neurodevelopmental disorders $[20,21]$. Since selection bias might have been produced by excluding the subjects without father registration, we also analyzed the group without father registration to assess this possibility. GWG below guidelines was significantly associated with a higher incidence of developmental delay than in the main analysis, although paternal medical history was not adjusted as a covariate in this subpopulation (Table S3). 
This study has several limitations. First, the data regarding developmental scores as measured by ASQ-3 were collected from parental self-reported questionnaires and therefore subjective. Parental self-reports are often retrospective and may include response bias from the emotionally sensitive nature of the questions. Accordingly, the diagnosis and severity of developmental delay were not definite. Second, as data on abnormalities were evaluated at 12 months of age, no neurodevelopmental disorders diagnosed afterwards were included. Third, the exclusion of participants with missing ASQ-3 data may have constituted selection bias. Fourth, the parental histories of neurodevelopmental disorders, epilepsy, and mental disease were also collected from self-reported questionnaires. Therefore, these answers might not have conformed to diagnostic criteria or ICD coding. Finally, the participants of this study were a large group of underweight mothers. Therefore, analysis of obesity and/or excessive GWG may be inadequate, but it can be a valuable and unique research that is impossible in other countries.

Despite the above limitations, this is the first investigation using a large dataset from a Japanese nationwide birth cohort study to examine the independent influence of insufficient GWG on offspring's neurodevelopment that controlled for confounders identified by previous reports including birth weight. This study indicates a need to reconsider the optimal BMI and GWG for women desiring pregnancy not only in Japan but also in other developed countries.

\section{Abbreviations}

ANOVA: analysis of variance

ASD: autism spectrum disorder

ASQ-3: Ages and Stages Questionnaire, third edition

BMI: body mass index

Cl: confidence interval

DD: developmental delay

DM/GDM: diabetes mellitus/gestational diabetes mellitus

GWG: gestational weight gain

HDP: hypertensive disorders of pregnancy

IOM: the Institute of Medicine

JECS: the Japan Environment and Children's Study

OR: adjusted odds ratio

SGA: small for gestational age 


\section{Declarations}

Funding Source: No funding was secured for this study.

Financial Disclosure: The JECS was funded by the Ministry of the Environment, Japan. The findings and conclusions contained in this article are solely those of the authors and do not represent the official views of the above government.

Conflicts of Interest: The authors declare no competing interests.

Availability of data: Data are unsuitable for public deposition due to ethical restrictions and legal framework of Japan. It is prohibited by the Act on the Protection of Personal Information (Act No. 57 of 30 May 2003, amendment on 9 September 2015) to publicly deposit the data containing personal information. Ethical Guidelines for Medical and Health Research Involving Human Subjects enforced by the Japan Ministry of Education, Culture, Sports, Science and Technology and the Ministry of Health, Labour and Welfare also restricts the open sharing of the epidemiologic data. All inquiries about access

to data should be sent to: jecs-en@nies.go.jp. The person responsible for handling enquiries sent to this email address is Dr Shoji F. Nakayama, JECS Programme Office, National Institute for Environmental Studies.

Code availability: Not applicable.

\section{Author's contributions:}

Dr. Motoki conceptualized and designed the study, carried out the analyses, drafted the initial manuscript.

Prof. Inaba conceptualized and designed the study, reviewed and revised the manuscript.

Dr. Shibazaki, Dr. Misawa. Dr. Ohira, Prof. Kanai, Prof. Kurita, Prof. Tsukahara, and Prof. Nomiyama designed the data collection instruments, collection data, and critically reviewed the manuscript for important intellectual content.

The JECS group reviewed the manuscript and provided critical advice.

All authors approved the final manuscript as submitted and agree to be accountable for all aspects of the work.

Ethics approval: The study received the approval of the $y$ the Institutional Review Board on Epidemiological Studies of the Ministry of the Environment as well as by the Ethics Committees of all participating institutions.

Consent to participate: All participants gave informed consent to participate. 
Consent for publication: All participants gave informed consent to publish data from the study

\section{Acknowledgments:}

The findings and conclusions of this article are solely the responsibility of the authors and do not represent the official views of the Ministry of the Environment. The authors would like to thank all participants of this study and all individuals involved in data collection, as well as Ms. Tomoko Kamijo for her assistance in data analysis and Mr. Trevor Ralph for his English editorial support.

\section{References}

1. Oberklaid F, Efron D (2005) Developmental delay-identification and management. Aust Fam Physician 34:739-742

2. Blumberg SJ, Bramlett MD, Kogan MD, Schieve LA, Jones JR, Lu MC (2013) Changes in prevalence of parent-reported autism spectrum disorder in school-aged U.S. children: 2007 to 2011-2012. Natl Health Stat Report (65):1-11

3. Boyle CA, Boulet S, Schieve LA et al (2011) Trends in the prevalence of developmental disabilities in US children, 1997-2008. Pediatrics 127:1034-1042. https://doi.org/10.1542/peds.2010-2989

4. Simeonsson RJ, Sharp MC (1992) Developmental delays. In: Hoekelman RA, Friedman SB, Nelson NM et al (ed) Primary pediatric care. Mosby-Year Book, St Louis, pp 867-870

5. Gottlieb CA, Maenner MJ, Cappa C, Durkin MS (2009) Child disability screening, nutrition, and early learning in 18 countries with low and middle incomes: data from the third round of UNICEF's Multiple Indicator Cluster Survey (2005-06). Lancet 374:1831-1839. https://doi.org/10.1016/s01406736(09)61871-7

6. Marchi J, Berg M, Dencker A, Olander EK, Begley C (2015) Risks associated with obesity in pregnancy, for the mother and baby: a systematic review of reviews. Obes Rev 16:621-638. https://doi.org/10.1111/obr.12288

7. Goldstein RF, Abell SK, Ranasinha S et al (2017) Association of gestational weight gain with maternal and infant outcomes: a systematic review and meta-analysis. JAMA 317:2207-2225. https://doi.org/10.1001/jama.2017.3635

8. Institute of Medicine and National Research Council Committee to reexamine IOM pregnancy weight guidelines; Rasmussen K, Yaktine AL (eds) (2009) Weight gain during pregnancy: reexamining the guidelines. Washington (DC): National Academic Press. https://doi.org/10.17226/12584

9. Press release from The Ministry of Labour Health and Welfare (2006) Optimal weight gain during pregnancy [translated from Japanese]. https://www.mhlw.go.jp/houdou/2006/02/dl/h0201-3a4.pdf

10. Shindo R, Aoki M, Yamamoto Y, Misumi T, Miyagi E, Aoki S (2019) Optimal gestational weight gain for underweight pregnant women in Japan. Sci Rep 9:18129. https://doi.org/10.1038/s41598-019$54550-y$ 
11. Duffany KO, McVeigh KH, Kershaw TS, Lipkind HS, Ickovics JR (2016) Maternal obesity: risks for developmental delays in early childhood. Matern Child Health J 20:219-230. https://doi.org/10.1007/s10995-015-1821-z

12. Windham GC, Anderson M, Lyall K et al (2019) Maternal pre-pregnancy body mass index and gestational weight gain in relation to autism spectrum disorder and other developmental disorders in offspring. Autism Res 12:316-327. https://doi.org/10.1002/aur.2057

13. Michikawa T, Nitta H, Nakayama SF et al (2018) Baseline profile of participants in the Japan Environment and Children's Study (JECS). J Epidemiol 2018;28:99-104. https://doi.org/10.2188/jea.je20170018

14. Ishitsuka K, Nakayama SF, Kishi R et al (2017) Japan Environment and Children's Study: backgrounds, activities, and future directions in global perspectives. Environ Health Prev Med 22:61. https://doi.org/10.1186/s12199-017-0667-y

15. Squires J, Bricker D (2009) Ages and Stages Questionnaires ${ }^{\circledR} і \mathrm{i}, \bigotimes$, Third Edition (ASQ-3 ${ }^{\mathrm{TM}}$ ). A parentcompleted child-monitoring system. Brookes Publishing Co. Baltimore, US

16. McDonald S, Kehler H, Bayrampour H, Fraser-Lee N, Tough S (2016) Risk and protective factors in early child development: results from the All Our Babies (AOB) pregnancy cohort. Res Dev Disabil 58:20-30. https://doi.org/10.1016/j.ridd.2016.08.010

17. Demirci A, Kartal M (2018) Sociocultural risk factors for developmental delay in children aged 3-60 months: a nested case-control study. Eur J Pediatr 177:691-697. https://doi.org/10.1007/s00431018-3109-y

18. Westgard C, Alnasser Y (2017) Developmental delay in the Amazon: The social determinants and prevalence among rural communities in Peru. PLoS One 12:e0186263. https://doi.org/10.1371/journal.pone.0186263

19. Sandin S, Lichtenstein P, Kuja-Halkola R, Larsson H, Hultman CM, Reichenberg A (2014) The familial risk of autism. JAMA 311:1770-1777. https://doi.org/10.1001/jama.2014.4144

20. Colvert $E$, Tick B, McEwen $F$ et al (2015) Heritability of autism spectrum disorder in a UK populationbased twin sample. JAMA Psychiatry 72:415-423. https://doi.org/10.1001/jamapsychiatry.2014.3028

21. Fujiwara K, Aoki S, Kurasawa K, Okuda M, Takahashi T, Hirahara F (2014) Associations of maternal pre-pregnancy underweight with small-for-gestational-age and spontaneous preterm birth, and optimal gestational weight gain in Japanese women. J Obstet Gynecol Res 40:988-994. https://doi.org/10.1111/jog.12283

22. Morisaki N, Nagata C, Jwa SC, Sago H, Saito S, Oken E, Fujikawa T (2017) Pre-pregnancy BMIspecific optimal gestational weight gain for women in Japan. J Epidemiol 27:492-498. https://doi.org/10.1016/j.je.2016.09.013

23. Nomura K, Nagashima K, Suzuki S, Itoh H (2019) Application of Japanese guidelines for gestational weight gain to multiple pregnancy outcomes and its optimal range in 101,336 Japanese women. Sci Rep 9:17310. https://doi.org/10.1038/s41598-019-53809-8 
24. Gardner RM, Lee BK, Magnusson C, Rai D, Frisell T, Karlsson H et al (2015) Maternal body mass index during early pregnancy, gestational weight gain, and risk of autism spectrum disorders: Results from a Swedish total population and discordant sibling study. Int J Epidemiol 44:870-883. https://doi.org/10.1093/ije/dyv081

25. Paljk I, Verdenik I, Blickstein I, Tul N (2021) Maternal BMI and weight gain in singleton pregnancies: has something changed in the last decade? J Matern Fetal Neonatal Med 34:7-11. https://doi.org/10.1080/14767058.2019.1570111

26. Francaite-Daugeliene M, Petrenko V, Baliutaviciene D, Velickiene D (2016) Retrospective analysis of age-adjusted body mass index among pre-pregnant women in the Lithuanian urban area during three decades. BMJ Open 6:e010927. https://doi.org/10.1136/bmjopen-2015-010927

27. Kubota K, Itoh H, Tasaka M et al, Hamamatsu Birth Cohort (HBC) Study Team (2013) Changes of maternal dietary intake, bodyweight and fetal growth throughout pregnancy in pregnant Japanese women. J Obstet Gynaecol Res 39:1383-1390. https://doi.org/10.1111/jog.12070

28. Uno K, Takemi Y, Hayashi F, Hosokawa M (2016) Nutritional status and dietary intake among pregnant women in relation to pre-pregnancy body mass index in Japan. Nihon Koshu Eisei Zasshi 63:738-749. Japanese

29. Vohr BR, Poggi Davis E, Wanke CA, Krebs NF (2017) Neurodevelopment: The impact of nutrition and inflammation during preconception and pregnancy in low-resource settings. Pediatrics 139(Suppl 1):S38-S49. https://doi.org/10.1542/peds.2016-2828f

30. Molloy AM, Kirke PN, Brody LC, Scott JM, Mills JL (2008) Effects of folate and vitamin B12 deficiencies during pregnancy on fetal, infant, and child development. Food Nutr Bull 29(2 supl):S101-S111;discussion S112-S115. https://doi.org/10.1177/15648265080292s114

31. Black MM (2008) Effects of vitamin B12 and folate deficiency on brain development in children. Food Nutr Bull 29(2 Supppl):S126-S131

32. Zeisel SH (2006) Choline: critical role during fetal development and dietary requirements in adults. Annu Rev Nutr 26:229-250. https://doi.org/10.1177/15648265080292s117

33. Anjos T, Altmäe S, Emmett P et al, NUTRIMENTHE Research Group (2013) Nutrition and neurodevelopment in children: focus on NUTRIMENTHE project. Eur J Nutr 52:1825-1842. https://doi.org/10.1007/s00394-013-0560-4

34. Wachs TD, Georgieff M, Cusick S, McEwen BS (2014) Issues in the timing of integrated early interventions: contributions from nutrition, neuroscience, and psychological research. Ann N Y Acad Sci 1308:89-106. https://doi.org/10.1111/nyas.12314

35. Sussman D, Ellegood J, Henkelman M (2013) A gestational ketogenic diet alters maternal metabolic status as well as offspring physiological growth and brain structure in the neonatal mouse. BMC Pregnancy Childbirth 13:198. https://doi.org/10.1186/1471-2393-13-198

36. Sussman D, Germann J, Henkelman M (2015) Gestational ketogenic diet programs brain structure and susceptibility to depression \& anxiety in the adult mouse offspring. Brain Behav 5:e00300. https://doi.org/10.1002/brb3.300 
37. Agarwal PK, Xie H, Sathyapalan Rema AS et al (2020) Evaluation of Ages and Stages Questionnaire (ASQ 3) as a developmental screener at 9, 18, and 24 months. Early Hum Dev 147:105081. https://doi.org/10.1016/j.earlhumdev.2020.105081

38. Fauls JR, Thompson BL, Johnston LM (2020) Validity of the Ages and Stages Questionnaire to identify young children with gross motor difficulties who require physiotherapy assessment. Dev Med Child Neurol 62:837-844. https://doi.org/10.1111/dmcn.14480

39. Ga HY, Kwon JY (2011) A comparison of the Korean ages and stages questionnaires and Denver developmental delay screening test. Ann Rehabil Med 35:369-374. https://doi.org/10.5535/arm.2011.35.3.369

40. Romero Otalvaro AM, Grañana N, Gaeto N et al (2018) ASQ-3: Validation of the Ages and Stages Questionnaire for the detection of neurodevelopmental disorders in Argentine children. Arch Argent Pediatr 116:7-13. https://doi.org/10.5546/aap.2018.eng

\section{Tables}




\begin{tabular}{|c|c|c|c|}
\hline Variable & $\begin{array}{l}\text { Normal } \\
\text { development }\end{array}$ & $\begin{array}{l}\text { Developmental } \\
\text { delay }\end{array}$ & $P$ value \\
\hline Participants, n & 19,751 & 10,943 & \\
\hline Prepregnancy BMI, kg/m² & $20.6(19.1,22.5)$ & $20.5(19.1,22.6)$ & $0.61 *$ \\
\hline Prepregnancy BMI group, n (\%) & & & 0.16 \\
\hline Underweight $(\mathrm{BMI}<18.5)$ & $2,995(15.2)$ & $1,735(15.9)$ & \\
\hline Normal weight (BMI 18.5-24.9) & $14,721(74.5)$ & $8,040(73.5)$ & \\
\hline Overweight (BMI 25.0-29.9) & $1,590(8.1)$ & $895(8.2)$ & \\
\hline Obese (BMI 30.0+) & $445(2.3)$ & $273(2.5)$ & \\
\hline Maternal GWG, kg & $10.4(8.1,12.8)$ & $9.9(7.7,12.2)$ & $\begin{array}{l}<0.001 \\
\star\end{array}$ \\
\hline Maternal GWG group, n (\%) & & & $<0.001$ \\
\hline Below & $11,567(58.6)$ & $6,960(63.6)$ & \\
\hline Within & $6,575(33.3)$ & $3,275(29.9)$ & \\
\hline Above & $1,609(8.1)$ & $708(6.5)$ & \\
\hline Maternal age at delivery, years & $31.0(28.0,34.0)$ & $32.0(29.0,35.0)$ & $\begin{array}{l}<0.001 \\
\star\end{array}$ \\
\hline Maternal age group, $\mathrm{n}(\%)$ & & & $<0.001$ \\
\hline$<35$ years & $13,647(69.1)$ & $6,816(62.3)$ & \\
\hline $35+$ years & $6,104(30.9)$ & $4,127(37.7)$ & \\
\hline Highest level of maternal education, $\mathrm{n}(\%)$ & & & $<0.001$ \\
\hline Junior high school & $735(3.7)$ & $285(2.6)$ & \\
\hline High school & $5,932(30.0)$ & $3,162(28.9)$ & \\
\hline Vocational school/Junior college & $8,670(43.9)$ & $4,696(42.9)$ & \\
\hline University/Graduate school & $4,414(22.3)$ & $2,800(25.6)$ & \\
\hline Annual household income, $\mathrm{n}(\%)$ & & & 0.001 \\
\hline$<4,000,000 \mathrm{JPY}$ & $7,796(39.5)$ & $4,098(37.4)$ & \\
\hline 4,000,000-7,999,999 JPY & $9,893(50.1)$ & $5,610(51.3)$ & \\
\hline $8,000,000+J P Y$ & $2,062(10.4)$ & $1,235(11.3)$ & \\
\hline Maternal smoking during pregnancy, $\mathrm{n}(\%)$ & $\begin{array}{l}741(3.8) \\
16 / 22\end{array}$ & $296(2.7)$ & $<0.001$ \\
\hline
\end{tabular}




\begin{tabular}{|c|c|c|c|}
\hline Partner's smoking during pregnancy, n (\%) & $8,657(43.8)$ & $4,155(38.0)$ & $<0.001$ \\
\hline Maternal drinking during pregnancy, $\mathrm{n}(\%)$ & $366(1.9)$ & $202(1.8)$ & 0.97 \\
\hline Maternal history of mental disease, $\mathrm{n}(\%)$ & $996(5.0)$ & $571(5.2)$ & 0.50 \\
\hline $\begin{array}{l}\text { Maternal history of developmental disorder, } \mathrm{n} \\
(\%)\end{array}$ & $5(0.03)$ & $9(0.08)$ & 0.046 \\
\hline Maternal history of epilepsy, n (\%) & $87(0.4)$ & $71(0.6)$ & 0.015 \\
\hline Partner's history of mental disease, $\mathrm{n}(\%)$ & $462(2.3)$ & $291(2.7)$ & 0.083 \\
\hline $\begin{array}{l}\text { Partner's history of developmental disorder, } n \\
(\%)\end{array}$ & $13(0.07)$ & $8(0.07)$ & 0.82 \\
\hline Partner's history of epilepsy, n (\%) & $72(0.4)$ & $51(0.5)$ & 0.19 \\
\hline \multicolumn{4}{|l|}{ Parity, n (\%) } \\
\hline Multiparous & $11,132(56.4)$ & $5,837(53.3)$ & $<0.001$ \\
\hline Means of pregnancy for current birth, $n(\%)$ & & & $<0.001$ \\
\hline Spontaneous & $18,548(93.9)$ & $10,043(91.8)$ & \\
\hline Ovulation induction through medication & $493(2.5)$ & $321(2.9)$ & \\
\hline Artificial insemination or in vitro fertilization & $710(3.6)$ & $579(5.3)$ & \\
\hline Maternal use of folic acid supplements, n (\%) & $422(2.1)$ & $276(2.5)$ & 0.030 \\
\hline $\begin{array}{l}\text { Diabetes mellitus/gestational diabetes } \\
\text { mellitus, } \mathrm{n}(\%)\end{array}$ & $542(2.7)$ & $337(3.1)$ & 0.091 \\
\hline Hypertensive disorder of pregnancy, $n(\%)$ & $503(2.5)$ & $355(3.2)$ & $<0.001$ \\
\hline Intrauterine growth restriction, n (\%) & $321(1.6)$ & $195(1.8)$ & 0.31 \\
\hline Mode of delivery for current birth, $\mathrm{n}(\%)$ & & & $<0.001$ \\
\hline Spontaneous delivery & $11,731(59.4)$ & $6,092(55.7)$ & \\
\hline Induced delivery & $3,649(18.5)$ & $1,926(17.6)$ & \\
\hline Vacuum extraction/Forceps delivery & $1,271(6.4)$ & $728(6.7)$ & \\
\hline Cesarean section & $3,100(15.7)$ & $2,197(20.1)$ & \\
\hline Gestational week & $39(38,40)$ & $39(38,40)$ & $\begin{array}{l}<0.001 \\
*\end{array}$ \\
\hline Gestational week, n (\%) & & & $<0.001$ \\
\hline$<37$ weeks & $296(1.5)$ & $258(2.4)$ & \\
\hline $37+$ weeks & $19,455(98.5)$ & $10,685(97.6)$ & \\
\hline
\end{tabular}




\begin{tabular}{|llll|}
\hline Birth weight, g & $3060(2832$, & $3010(2776,3256)$ & $<0.001$ \\
& $3308)$ & & $<0.001$ \\
\hline Birth weight, $\mathrm{n}(\%)$ & & $0(0)$ & \\
\hline$<1500$ & $0(0)$ & $771(7.0)$ & \\
\hline 1500 to $2499 \mathrm{~g}$ & $949(4.8)$ & $10,172(93.0)$ & \\
\hline $2500+\mathrm{g}$ & $18,802(95.2)$ & $5,930(54.2)$ & $<0.001$ \\
\hline Gender (male), $\mathrm{n}(\%)$ & $9,506(48.1)$ & & \\
\hline Method of feeding, $\mathrm{n}(\%)$ & & $5,662(51.7)$ & \\
\hline Breast feeding & $11,147(56.4)$ & $4,763(43.5)$ & \\
\hline Mixed feeding & $7,889(39.9)$ & $418(3.8)$ & 0.001 \\
\hline Infant formula & $583(3.0)$ & $100(0.9)$ & \\
\hline Other & $132(0.7)$ & $1,593(14.6)$ & \\
\hline Neonatal jaundice, $\mathrm{n}(\%)$ & $2,639(13.4)$ & & \\
\hline
\end{tabular}

BMI denotes body mass index, GWG gestational weight gain.

* Mann-Whitney U test between Normal development vs. Developmental delay. 
Table 2. ASQ-3 domain scores and proportions at risk of delay according to maternal gestational weight gain

ASQ-3 domain (cut-off score,)

$\begin{array}{llll}\text { Below } & \text { Within } & \text { Above } & \text { P } \\ n=18,527 & n=9,850 & n=2,317 & \text { value }\end{array}$

Communication (15.64 points)

score

$37.3 \pm 13.4$

$38.7 \pm 13.2$

$40.3 \pm 13.0$

$<$

On track, n (\%)

$17,141(92.5)$

$9,266(94.1)$

$1,386(7.5)$

$584(5.9)$

2,211 (95.4)

Referral, n (\%)

$42.4 \pm 17.5 \quad 44.0 \pm 16.7$

$45.1 \pm 16.5$

$<$

score

15,833 (85.5)

8,652 (87.8)

2,056 (88.7)

Referral, n (\%)

2,694 (14.5)

$1,198(12.2)$

261 (11.3)

$<$

0.001

Fine motor (34.50 points)

score

$48.0 \pm 11.5$

$49.0 \pm 11.0$

$49.8 \pm 10.6$

$<$

0.001 *

On track, n (\%)

16,600 (89.6)

$8,977(91.1)$

$2,147(92.7)$

Referral, n (\%)

$1,927(10.4)$

873 (8.9)

170 (7.3)

$<$

0.001

Problem solving (27.32 points)

scores

$42.2 \pm 13.4$

$43.1 \pm 13.2$

$43.8 \pm 13.0$

$<$

On track, n (\%)

$15,633(84.4)$

$8,437(85.7)$

2,023 (87.3)

Referral, n (\%)

2,894 (15.6)

1,413 (14.3)

$294(12.7)$

$<$

0.001

Personal-social (21.73 points)

\begin{tabular}{lllll} 
scores & $36.6 \pm 14.4$ & $38.2 \pm 14.1$ & $39.4 \pm 14.0$ & $<.001$ * \\
\hline On track, n (\%) & $15,225(82.2)$ & $8,399(85.3)$ & $2,006(86.6)$ & \\
\hline Referral, n (\%) & $3,302(17.8)$ & $1,451(14.7)$ & $311(13.4)$ & $<$ \\
& & & & 0.001
\end{tabular}


Plus-minus variables are means \pm standard deviation.

* Differences in scores of ASQ-3 domain were assessed with one-way repeated measures of ANOVA.

Table 3. Odds ratio and $95 \%$ confidence intervals for the association between gestational weight gain (GWG) categories and developmental delay in ASQ-3 domains

\begin{tabular}{|c|c|c|c|c|c|c|}
\hline & $\begin{array}{l}\text { Within GWG } \\
\text { (reference) }\end{array}$ & Below GWG & & Above GWG & & $\begin{array}{l}\text { Every } \\
2.3 \mathrm{~kg}(5 \mathrm{lb}) \\
\text { increased }\end{array}$ \\
\hline & $\begin{array}{l}\text { No. cases / } \\
\text { Normal } \\
\text { development }\end{array}$ & $\begin{array}{l}\text { No. cases / } \\
\text { Normal } \\
\text { development }\end{array}$ & $\begin{array}{l}\text { OR } \\
(95 \% \\
\mathrm{Cl})\end{array}$ & $\begin{array}{l}\text { No. cases / } \\
\text { Normal } \\
\text { development }\end{array}$ & $\begin{array}{l}\text { OR } \\
(95 \% \\
\mathrm{Cl})\end{array}$ & $\begin{array}{l}\mathrm{OR}(95 \% \\
\mathrm{Cl})\end{array}$ \\
\hline \multicolumn{7}{|l|}{ ASQ-3 domains } \\
\hline Communication & $584 / 6575$ & $1386 / 11567$ & $\begin{array}{l}1.21 \\
(1.09- \\
1.34)\end{array}$ & $106 / 1609$ & $\begin{array}{l}0.82 \\
(0.66- \\
1.03)\end{array}$ & $\begin{array}{l}0.91(0.88 \\
0.94)\end{array}$ \\
\hline Gross motor & $1198 / 6755$ & $2694 / 11567$ & $\begin{array}{l}1.14 \\
(1.05- \\
1.24)\end{array}$ & $261 / 1609$ & $\begin{array}{l}0.98 \\
(0.84- \\
1.13)\end{array}$ & $\begin{array}{l}0.96(0.94 \\
0.98)\end{array}$ \\
\hline Finte motor & $873 / 6575$ & $1927 / 11567$ & $\begin{array}{l}1.13 \\
(1.04- \\
1.24)\end{array}$ & $170 / 1609$ & $\begin{array}{l}0.84 \\
(0.70- \\
1.00)\end{array}$ & $\begin{array}{l}0.94(0.91 \\
0.96)\end{array}$ \\
\hline $\begin{array}{l}\text { Problem } \\
\text { solving }\end{array}$ & $1413 / 6575$ & $2894 / 11567$ & $\begin{array}{l}1.09 \\
(1.01- \\
1.18)\end{array}$ & $294 / 1609$ & $\begin{array}{l}0.85 \\
(0.74- \\
0.98)\end{array}$ & $\begin{array}{l}0.95(0.93 \\
0.97)\end{array}$ \\
\hline Personal-social & $1451 / 6575$ & $3302 / 11567$ & $\begin{array}{l}1.15 \\
(1.07- \\
1.24)\end{array}$ & $311 / 1609$ & $\begin{array}{l}0.94 \\
(0.82- \\
1.08)\end{array}$ & $\begin{array}{l}0.94(0.92 \\
0.96)\end{array}$ \\
\hline
\end{tabular}

OR adjusted odds ratio, $\mathrm{Cl}$ confidence interval, and GWG gestational weight gain, BMI body mass index, DM/GDM diabetes mellitus/gestational diabetes mellitus, and HDP hypertensive disorder of pregnancy.

These ORs were adjusted for maternal age, prepregnancy BMI, parental smoking habit, maternal drinking habit, maternal highest level of education, annual household income, parental history of developmental disorders, epilepsy, and mental disease, means of pregnancy, use of folic acid supplements, complications during pregnancy (including DM/GDM, and HDP), intrauterine growth restriction, gender, birth weight, method of feeding, and neonatal jaundice.

\section{Figures}


3,921 Excluded

1,254 Miscarriages

382 Stillbirths

2,285 Missing data on pregnancy

100,144 Live births

1,889 Excluded due to multiple births

1,845 Twin, 44 Triplets

98,255 Singleton live births

47,692 No registration of fathers

50,563 Singleton live births with

participating fathers

9,053 Missing score in one or more of the five domains in

the ASQ-3 questionnaires

7,821 Missing data for analysis

33,689 Eligible live births

2,995 Excluded

144 Infants with low Apgar score of 5 minutes

( $<7$ points)

2,663 Infants with some physical abnormality

32 Infants diagnosed as having congenital metabolic disorder by screening test

156 Infants with abnormality in hearing ability screening test

30,694 Analyzed live births

Male: 15,436, Female: 15,258

Figure 1

Case selection flowchart 

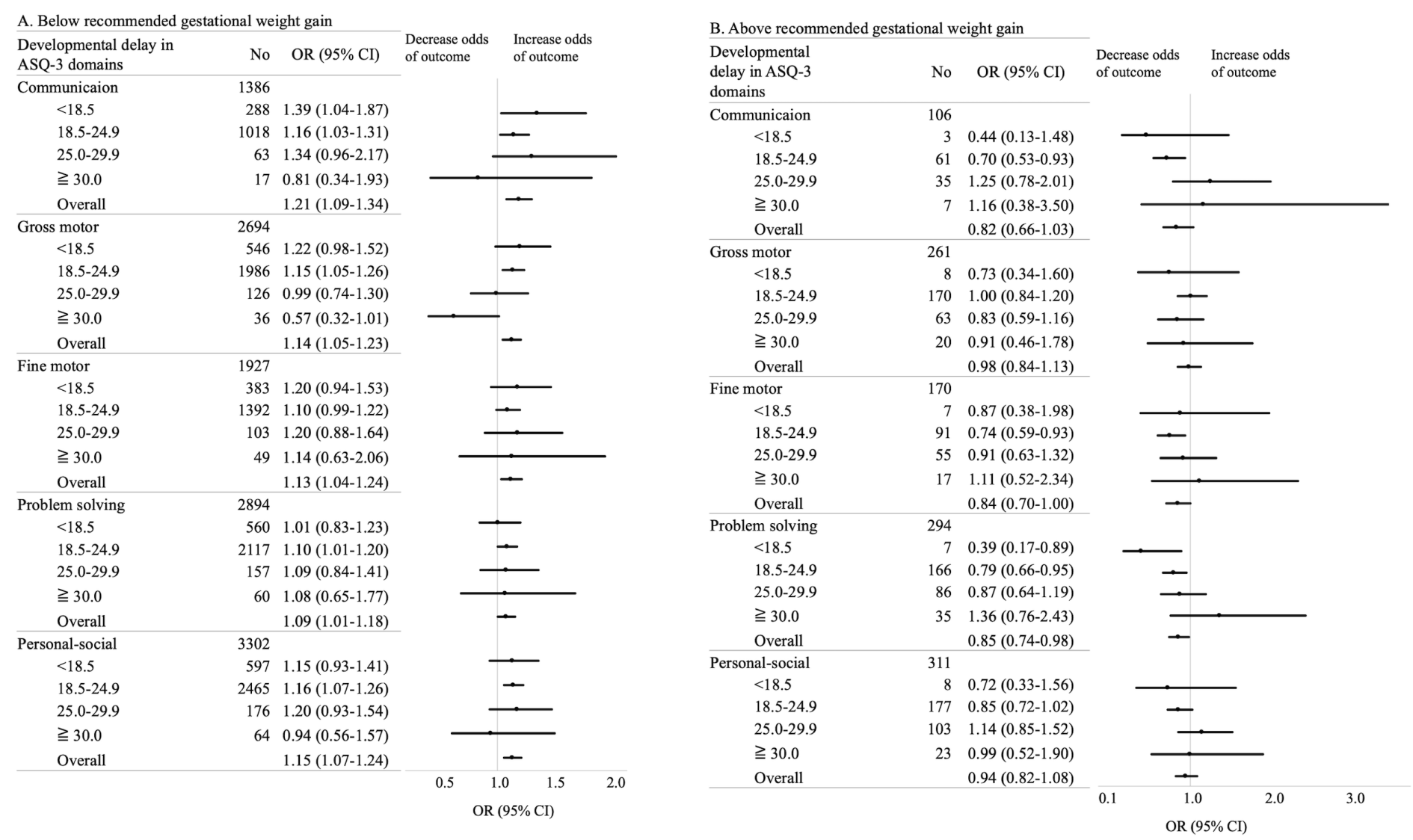

Figure 2

Odds ratios (ORs) for the association between gestational weight gain (GWG) below and above guidelines with developmental delay in ASQ-3 domains according to the prepregnancy body mass index (BMI) categories ORs are shown for the association between GWG below (A) and above (B) guidelines with developmental delay in ASQ-3 domains. Reference group is mothers with recommended weight gain in each category of prepregnancy BMI. These ORs were adjusted for maternal age, prepregnancy BMI, parental smoking habit, maternal drinking habit, maternal highest level of education, annual household income, parental history of developmental disorders, epilepsy, and mental disease, means of pregnancy, use of folic acid supplements, complications during pregnancy (including DM/GDM, and HDP), intrauterine growth restriction, gender, birth weight, method of feeding, and neonatal jaundice.

\section{Supplementary Files}

This is a list of supplementary files associated with this preprint. Click to download.

- MotokiNSupplementalTables.pdf 